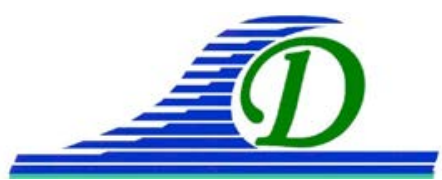

XIII ${ }^{\text {èmes }}$ Journées Nationales Génie Côtier - Génie Civil

Dunkerque, 2-4 juillet 2014

DOI:10.5150/jngcgc.2014.096 @ Editions Paralia CFL

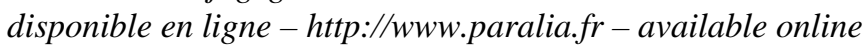

\title{
Retour d'expérience de deux cas de dépoldérisation confronté au projet de gestion du littoral des bas-champs de Cayeux/Mer (Picardie)
}

\author{
Vincent BAWEDIN ${ }^{1}$
}

\author{
1. Conseil général de la Somme, Direction Générale Adjointe de l’Aménagement et de \\ l’Equipement - Mission "Stratégie-Littoral", \\ 43 rue de la République, 80026 Amiens cedex 1, France. \\ v.bawedin@somme.fr
}

\section{Résumé :}

La dépoldérisation, qui consiste au retour de la mer sur des terres jadis conquises, est encore timorée en France alors qu'elle se développe en Angleterre, en Allemagne ou aux Pays-Bas. Dans l'hexagone, de tels phénomènes sont le plus souvent subis, résultant de tempêtes, d'inondations, et non pas choisis, contrairement aux cas anglo-saxons. Il a été jugé opportun de revenir sur deux cas de dépoldérisations qui répondent à la volonté des pouvoirs publics, dont un à des fins de défense contre les risques de submersions marines (cas anglais, pour lequel nous pourrions parler de "dépoldérisation offensive à des fins défensives").

Ces retours d'expériences seront confrontés à l'étude en cours de dépoldérisation partielle, éventuelle et maîtrisée qui concerne aujourd'hui le trait de côte des baschamps de Cayeux/Mer (Somme), coordonnée par le bureau d'études Artelia afin de sonder la faisabilité d'une telle opération à des fins de défense contre les risques. Directement inspirée de cas anglais, cette initiative, quel qu'en soit l'aboutissement, est une première dans l'hexagone et mérite à ce seul titre d'être analysée.

Elle est menée parallèlement aux travaux entrepris sur la digue de galets au sud de la baie de Somme (24 épis en cours de construction, dans la continuité de 80 déjà existant) montrant que prédomine encore une lutte continue contre l'élément marin, relevant de la protection ou de la consolidation suite à des tempêtes. Le bassin d'Arcachon, en Aquitaine, possède déjà quant à lui plusieurs sites rendus à la mer, même si ce choix de gestion n’a pas été motivé par la lutte contre les effets de la hausse eustatique, contrairement au cas anglais présenté et à ce qui a motivé l'étude picarde.

Néanmoins, un changement sensible semble s'opérer en France, avec l'émergence de solutions plus douces voire même audacieuses comme nous le montre l'exemple picard où la poursuite des épis a été conditionnée à l'étude prospective d'une possible dépoldérisation en amont dérive sur une surface de plus de 1000 hectares. Celle-ci, décidée par le Conseil général de la Somme, sous maîtrise d’ouvrage du Syndicat Mixte Baie de Somme Grand Littoral Picard, rendra ses conclusions fin 2014. 


\section{Thème 7 - Risques côtiers}

Mots clés : Gestion du trait de côte, Effets du changement climatique, Dépoldérisation, Gouvernance, Approche anglo-saxonne, Centralisme français, Initiatives novatrices, Développement durable, Gestion Intégrée des Zones Côtières (GIZC), Prospective.

Key-words: Coastline management, Effects of climate change, Managed realignment, Planning coastal policies, Governance, Anglo-saxon approach, French centralism, Innovative project, Sustainable developement, Integrated Coastal Zone Management (ICZM), Prospective.

\section{Introduction}

Depuis les XVII ${ }^{\mathrm{e}}$ et XVIII ${ }^{\mathrm{e}}$ siècles, sous l'influence des ingénieurs hollandais, la gestion visant à la stabilité du trait de côte en France a caractérisé une grande ambition des hommes (PASKOFF, 1994), avec leurs moyens techniques, pour influer sur ce qui apparaît aujourd'hui de plus en plus comme "un phénomène dynamique impossible à fixer une fois pour toute" (VEYRET, 2004), situation confirmée par les tempêtes de début 2014 et leurs conséquences sur le recul inéluctable de certains secteurs de la côte aquitaine, par exemple. Cette conception "fixiste" de l'aménagement du littoral, chère au corps d'ingénieurs français dont on connaît la vision anthropocentrique, répond souvent à une volonté de planification rationnelle de l'Etat, acteur longtemps exclusif sur le littoral. Les approches anglo-saxonnes diffèrent en la matière, se montrant plus globales et davantage inclinées à la prise en compte du long terme. En ce sens, elles répondent mieux aux principes de développement durable et de Gestion Intégrée des Zones Côtières (GIZC), gouvernance inspirée des expériences européenne et plus précisément d'Europe du Nord-Ouest.

Les événements récents (Xynthia en 2010, Petra en 2014), associés à la prise de conscience des conséquences du changement climatique déjà observables (voir $5^{\mathrm{e}}$ rapport du GIEC), semblent faire émerger une nouvelle approche en termes de gestion des risques littoraux, et par conséquent, d’aménagement du littoral.

\section{Matériel et méthode}

La démarche ici n'est pas celle, technique, d'un ingénieur mais davantage une analyse de géographe ayant réalisé sa thèse sur la GIZC et en même temps de praticien et aménageur du littoral dans une collectivité investie dans plusieurs études de dépoldérisation. La méthode est donc une démarche holistique, une comparaison de cas observés in situ dans le cadre de travaux de recherche ou de projets comme celui de la

dépoldérisation des bas-champs de Cayeux, où la prospective tient une place importante.

\section{Résultats}

C’est en Allemagne que le phénomène de dépoldérisation a été amorcé, les Pays-Bas et l'Angleterre ont ensuite suivi. On sait pourtant à quel point la lutte contre l'élément 


\section{XIII ${ }^{\text {èmes }}$ Journées Nationales Génie Côtier - Génie Civil \\ Dunkerque, 2-4 juillet 2014}

marin est inscrite dans l'histoire des Néerlandais, dont beaucoup ont encore en mémoire la catastrophe de 1953 qui a entraîné la mort de 1850 personnes ainsi que 300 victimes en Angleterre. Nous abordons ici un exemple de dépoldérisation par création de brèche(s) en Angleterre effectué à des fins de protection de la population contre la mer puis un cas concernant le commune de La Teste de Buch dans le bassin d'Arcachon. Ce dernier, pour lequel il vaudrait mieux parler de "remaritimisation" que de dépoldérisation, n’a aucune prétention à amoindrir les risques de submersion, mais présente un intérêt d'un autre ordre.

Enfin, l'étude concernant les bas-champs de Cayeux/mer, site particulièrement exposé à l'érosion, est inspirée des cas de dépoldérisations à des fins de protection tel que le cas anglais, ce dernier ayant cependant été réalisé sur un littoral en accrétion. Elle vise à proposer des solutions pérennes de gestion d'un trait de côte qui ne soient plus tributaires de stratégies plus "lourdes", dépendantes de rechargements artificiels en galets, ressource épuisable.

\subsection{Le cas de Tollesbury (Essex) : une dépoldérisation défensive par brèche}

Le schorre protège plus de 66\% des côtes de l'Essex. Il a été jugé opportun de le protéger afin de l'utiliser comme moyen de défense contre la mer, ce qui permet de cumuler des raisons écologiques (les marais maritimes présentent une riche biodiversité) et de protection côtière. Le polder de Tollesbury a ainsi été remis en contact avec la mer en 1995, par la création d'une brèche, large d'une dizaine de mètres (photo 1). Ce programme a été co-lancé par English Nature et le ministère de l'Agriculture (MAFF) en 1993 afin de repenser les techniques de défense contre la mer.

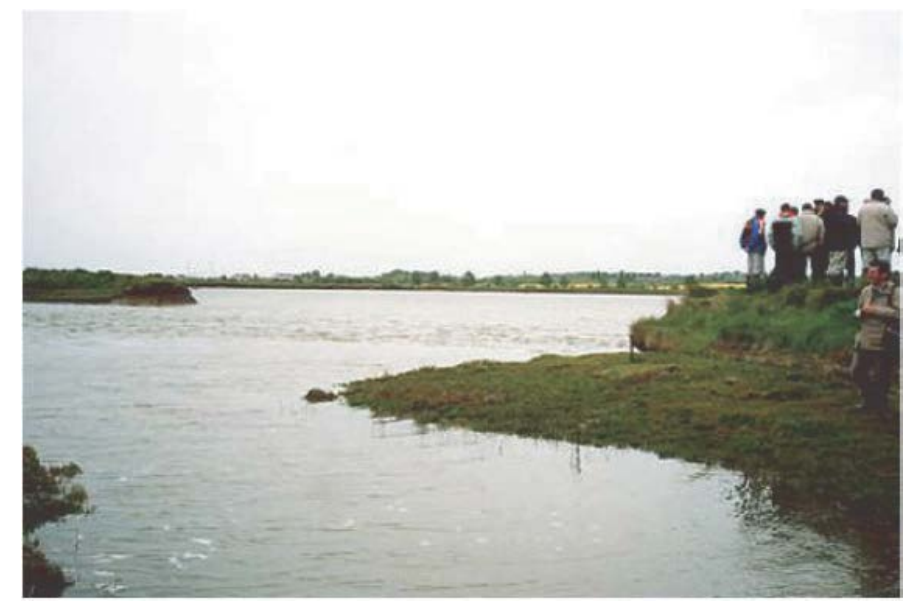

Photo 1. Dépoldérisation par ouverture de brèche dans la digue une gestion qui a fait ses preuves en Angleterre en termes de lutte contre les effets de la hausse du niveau des mers (Tollesbury, V. Bawedin, mai 2003). 


\section{Thème 7 - Risques côtiers}

Une digue, haute seulement de deux à trois mètres, a néanmoins été édifiée en arrière afin de protéger les terres agricoles de l'arrière pays. Ainsi, à l'endroit de l'ancienne portion de digue retirée et à l'avant de la nouvelle, édifiée en retrait, s'est développé, grâce à l'accumulation de sédiments marins, un haut schorre. Comme le relate la géographe Lydie GOELDNER (1999), des études effectuées par la National River Authorities (NRA) montrent qu'en l'absence de schorre, la digue, pour être efficace, doit avoir une hauteur de 12 mètres ; ce qui entraînerait un coût de 5000 livres par mètre linéaire. En revanche, grâce à la présence d'un schorre dont la surface aura été multiplié par sept, la hauteur de la digue peut s'abaisser de six à trois mètres, pour des coûts respectifs de 1500 et 400 livres par mètre linéaire (GOELDNER, op. cit. ; BAWEDIN, 2004). Cela revient à réaliser jusqu’à dix fois plus d'économie.

Cette dépoldérisation a permis l'apparition de plantes pionnières caractéristiques de la slikke, telles Salicornia obscura, qui ont recolonisé le polder, deux ans seulement après sa réouverture. Cela traduit la renaissance d'un marais maritime complet, synonyme d'extension du schorre. Le but ici était double : favoriser le retour du schorre, en voie de raréfaction en Angleterre étant données les poldérisations nombreuses qui ont eu lieu, et expérimenter un retrait contrôlé pour évaluer les potentialités des prés salés à défendre les côtes contre les effets induits de l'élévation de la mer. Cette technique de retrait, bénéfique tant d'un point de vue écologique qu'humain et économique, est maîtrisée et contrôlée par les aménageurs, d'où son nom de "retrait contrôlé", ou encore de managed realignment (GOELDNER-GIANELLA, 2008). Ce cas anglais pourrait-il être "extrapolé" en France?

\subsection{Les Prés salés ouest de La Teste (Aquitaine) rendus à la mer par vannes}

Un autre cas de retour programmé de la mer est l'un de ceux concernant le bassin d'Arcachon : les Prés salés ouest de La Teste de Buch (BAWEDIN, 2013).

En 2008, les prés salés sont remis en eau, par un système de vannes construites dans la digue séparant les prés salés du bassin d'Arcachon (photo 2). L’objectif ici n’est cependant pas la lutte contre les risques mais une volonté de renaturation d'un espace peu ou prou abandonné qui n’avait plus de "prés salés" que le nom. Il a d’abord résulté de cette opération récente non pas le retour d'un caractère maritime mais d'un marais d'eau douce à saumâtre, avec développement de phragmitaies (roselières) inondées, propices à l'apparition d'une avifaune paludicole typique. Cette évolution s'explique par la présence de nombreux canaux d'eau douce, appelés crastes localement, qui alimentent également les prés salés. La gestion de ces canaux alimentant l'espace concerné (photo 3) peut être un moyen de rouvrir à la mer tout en maintenant ou favorisant si besoin (raisons écologiques) le développement d'espèces liées au milieu d'eau douce. 


\section{XIII ${ }^{\text {èmes }}$ Journées Nationales Génie Côtier - Génie Civil \\ Dunkerque, 2-4 juillet 2014}

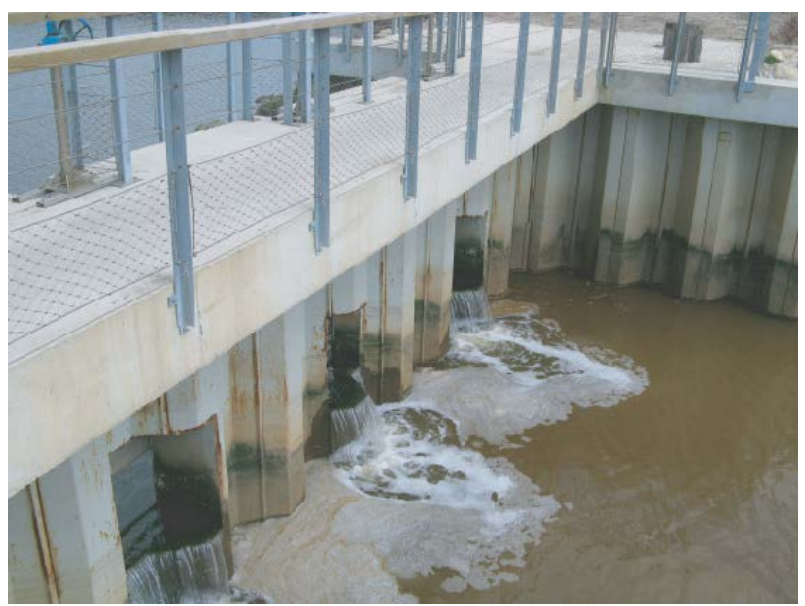

Photo 2. Barrage avec vannes permettant l'entrée de l'eau de mer à marée haute. Il est situé sur la digue séparant le bassin d'Arcachon des prés salés Ouest (La Teste de Buch, V. Bawedin, avril 2010).

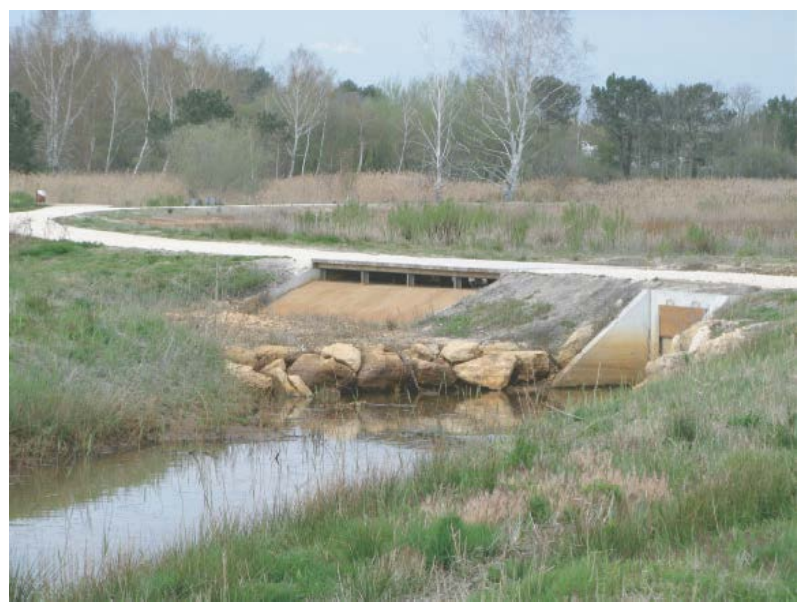

Photo 3. Craste alimentant par une vanne la zone rendue à la mer et ralentissant le développement d'un milieu halophile. Une partie de la roselière est ici visible, derrière le chemin de découverte (La Teste de Buch, V. Bawedin, avril 2010).

3.3 Les bas-champs de Cayeux/Mer (Picardie) : protection par épis et perspective de réouverture

Les Bas-champs de Cayeux, situés au sud de la baie de Somme, constituent un espace protégé de la mer par un cordon de galets (figure 1) entretenu à grands frais.

Ils ont été clos à la mer par le "Grand Barrement" au milieu du XVIII ${ }^{\mathrm{e}}$ siècle. La partie qui s'étend de Ault-Onival jusqu'à Cayeux-sur-Mer (soit environ 7 kilomètres) et, dans une moindre mesure, au niveau du poulier du Hourdel, est concernée par l'érosion (DOLIQUE \& ANTHONY, 1999). Cela fait près de trois siècles que le combat dure contre la mer. Des brèches se sont ouvertes, au sud - sud-ouest de Cayeux, lors de fortes marées coïncidant avec des vents d'ouest soutenus et importants. L'inondation la plus 


\section{Thème 7 - Risques côtiers}

importante date de février 1990, résultant de la concomitance de paramètres météorologiques exceptionnels et de période de marées à forts coefficients. Le coût des travaux pour le maintien et la consolidation de la digue tout en respectant le caractère naturel du cordon (rechargement de galets, casiers, épis), qui s’apparente à un matelas protecteur, s'élève, depuis la tempête de 1990, à plus de 40 millions d'euros auxquels il convient d'ajouter près de 350000 euros par an pour l'entretenir (BAWEDIN \& HOEBLICH, 2006). Il importe de préciser que cette solution relativement "souple" a été choisie tandis que d'autres, plus dures, tels les enrochements, ont été avancées. Ces derniers auraient eu pour conséquence d'empêcher le transit sédimentaire donc de priver la digue de sa ressource en galets qui proviennent de l'érosion des falaises situées en amont dérive. Le choix de gestion des pouvoirs publics concernant ce cordon de galets est cependant emblématique de "l'acharnement thérapeutique", d'autant plus que les coûts investis pour la protection de cette digue servent à protéger un espace non urbanisé.

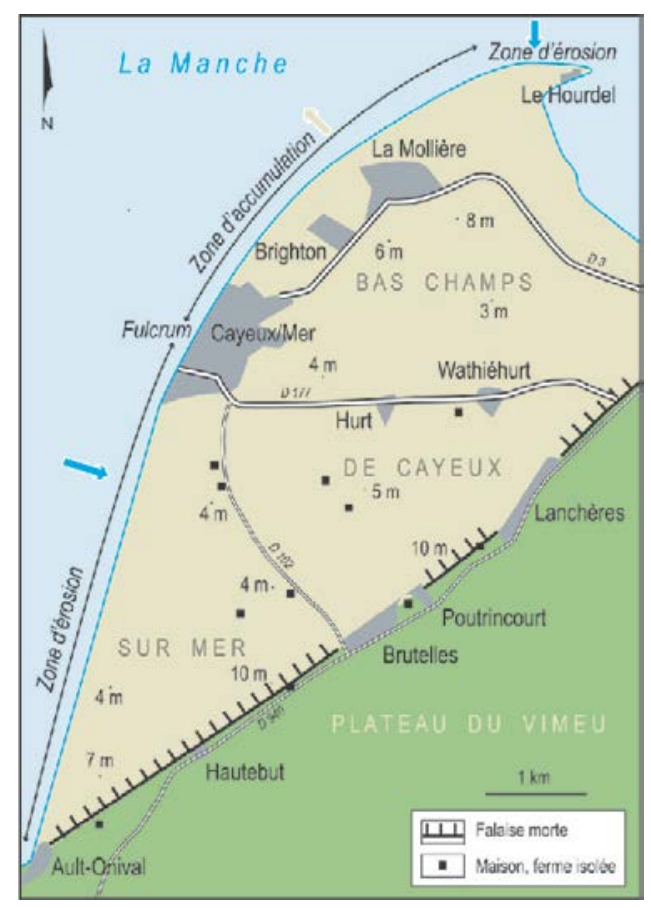

Figure 1. Carte des bas-champs de Cayeux/Mer. Le trait de côte érodé se situe au sud de la ville de Cayeux/Mer. 80 épis ont été construits et 24 sont en cours de construction devant Cayeux/Mer. Si ces derniers protégent la ville face à un risque frontal de submersion, l'hypothèse de dépoldériser au sud est envisagée afin de reconstituer, au sud de Cayeux, une zone dite "zone tampon" qui pourrait jouer un rôle naturel de protection (carte : LGPA in BAWEDIN, 2012). 


\section{XIII ${ }^{\text {èmes }}$ Journées Nationales Génie Côtier - Génie Civil \\ Dunkerque, 2-4 juillet 2014}

\subsubsection{L'installation des épis se poursuit : 24 nouveaux installés en 2014}

Les pouvoirs publics (Etat, collectivités) ont signé une convention, en 2012, dans laquelle ils s'engagent à réaliser 24 nouveaux épis qui seront installés sur le front de mer de Cayeux (+ 1 kilomètre). Les travaux seront achevés début 2015, avec un rechargement, à l'image de ce qui existe déjà en amont dérive. Le coût global de l'opération s'élève à 18 millions d'euros. Nous avons néanmoins à faire à une nouvelle approche du problème, à la fois concrète et pragmatique, et prenant en compte les dynamiques naturelles. En effet, cette création d'épis s'accompagne parallèlement d'une étude sur la faisabilité d'une dépoldérisation partielle des Bas champs à des fins défensives, ce qui constitue une première en France pour un site aussi exposé. Les deux initiatives ne sont pas contradictoires mais au contraire complémentaires. Les 24 épis sont installés sur le front de mer de Cayeux (zone du fulcrum sur la figure 1) afin de protéger la ville d'assauts frontaux. En revanche, l'étude de dépoldérisation concerne une zone située en amont-dérive (où se trouvent les 80 premiers épis), concernée par l'érosion que tentent de palier les dépenses de travaux et rechargements somptuaires entrepris depuis plusieurs décennies. Cette étude est entièrement financée par l'Etat, avec l'aide de crédits européens (Feder, Dreal, Agence de l'Eau Artois Picardie). Quelle que soit la décision finale des pouvoirs publics (rouvrir ou non), la présence des 24 épis s'avèrera indispensable.

\subsubsection{Cayeux-sur-mer : vers une gestion inspirée du modèle anglo-saxon ?}

A ce jour, aucun exemple français de dépoldérisation ne correspond à une volonté de lutte contre les risques de submersion et/ou les effets de l'élévation du niveau de la mer, tel que cela est pratiqué en Grande Bretagne. Pourtant, les cas anglais le montrent, dépoldériser permet, par recréation de marais maritimes, l'apport de sédiments et de plantes halophiles qui, au grès du temps et des marées, reconstitueront une véritable zone de haut schorre susceptible de jouer le rôle de frein contre les assauts des vagues.

L'une des explications de cette réticence réside peut-être dans le fait qu'une gestion littorale utilisant la dépoldérisation revient à choisir de laisser passer ou "laisser-faire" l'élément marin sur des terres gagnées de haute lutte du $\mathrm{XV}^{\mathrm{e}}$ au XVIII ${ }^{\mathrm{e}}$ siècles. Or le laisser-faire, qui pourrait s'apparenter à un "libéralisme écologique" (BAWEDIN, 2004) est généralement aux antipodes de la gestion, qui consiste à agir, à maîtriser et ne s'accommode pas de "main invisible" pour transposer cette métaphore économique au domaine de l'aménagement du territoire. Quatre scénarii proposant une ouverture par brèche d'ampleur différente ont été étudiés par Artelia. Grace à une modélisation, l'espace recouvert par les marées en fonction des coefficients, la vitesse et la fréquence de l'entrée de l'eau de mer ont été calculés. Le choix retenu par le comité de pilotage, c'est-à-dire la solution la plus à même de protéger le plus efficacement Cayeux/Mer par le Sud-Ouest, est le scénario $n^{\circ} 3$ (figure 2) qui propose une réouverture de 1045 hectares grâce à une brèche envisagée initialement longue de 440 mètres mais qui 


\section{Thème 7 - Risques côtiers}

devrait probablement dépasser 600 mètres, ce afin de ne pas créer un épi hydraulique eu égard à la dynamique littorale orientée du sud vers le nord.

L'efficacité d'une telle initiative est étudiée par plusieurs paramètres dont plusieurs ont été modélisés : la vitesse à laquelle se développerait un marais maritime susceptible de freiner les vagues et la capacité de la mer à occuper l'espace ouvert sans que cela ne soit problématique. Le risque de ne pas contrarier la dynamique littorale par l'épis hydraulique que représenterait la brèche (ce qui justifie d'autant plus, le cas échéant, la présence de 24 épis en aval dérive). La nature possible des usages après dépoldérisation, au travers de données sur le nombre de jours par an où la mer serait présente (et avec quelle hauteur d'eau) en fonction des coefficients de marées. La localisation d'arrièresdigues à rehausser, si nécessaire, et avec quelle ampleur. Et bien évidemment, le coût d'une telle initiative et sa faisabilité au regard des divers et nombreux outils juridiques qui s'appliquent à ce territoire littoral.

Les pouvoirs publics auront fin 2014 les éléments qui leur permettront de prendre, le moment venu, une décision éclairée par cette étude, sur une stratégie nouvelle qui n'avait jusqu'alors jamais été menée en France sur un secteur côtier en érosion, sur de telles surfaces, et pour répondre au risque de submersion. C’est aujourd'hui chose faite.

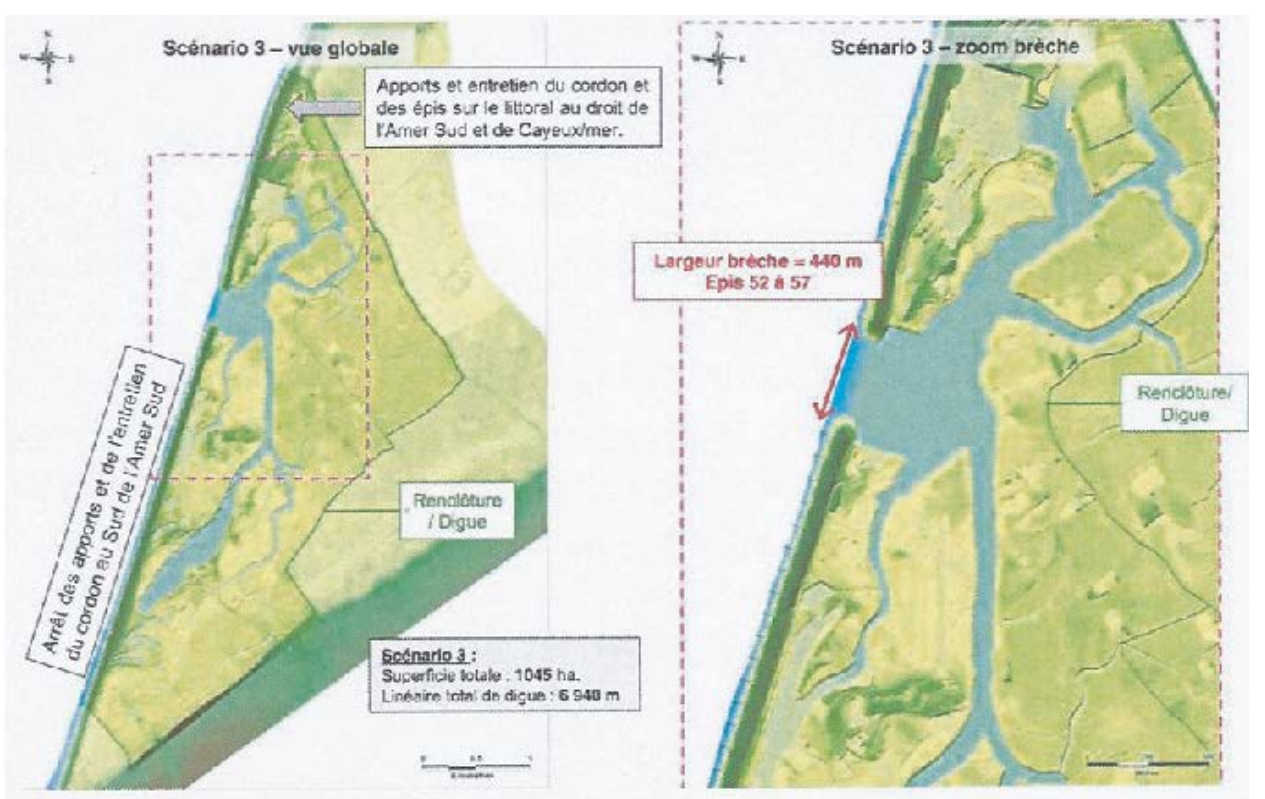

Figure 2. Schéma d'ouverture du cordon de galets, scénario 3 (ARTELIA, 2013).

\section{Conclusion}

La dépoldérisation utilisée comme moyen défensif contre les submersions marines est une technique efficiente en Angleterre depuis une quinzaine d'années.

En France, la dépoldérisation, plus timorée, a été subie davantage que choisie puisque répondant le plus souvent à une volonté de maintenir la mer sur des sites qu'elle a 


\section{XIII ${ }^{\text {èmes }}$ Journées Nationales Génie Côtier - Génie Civil \\ Dunkerque, 2-4 juillet 2014}

réoccupés après une tempête. Rares sont les choix d'un retour programmé de la mer résultant de la volonté des pouvoirs publics. A ce jour, les Prés salés ouest de la Teste de Buch font quasiment figure d'exception. Il faudra ajouter, dès 2016, le cas de la Ferme de La Caroline en baie de Somme, qui répond à des objectifs hydrauliques (maintien du caractère maritime) et n’a donc pas été présenté ici. Mais aucun cas français ne répond à une tactique de lutte contre les effets de la hausse eustatique. C'est pourquoi le projet étudié dans les Bas-champs de Cayeux est une première et dénote une approche innovante, même si leur dépoldérisation n'en est qu'au stade de l'hypothèse.

Nous sommes toutefois là dans la droite ligne de la nouvelle stratégie de l'Etat en matière de gestion du littoral, celui-ci incitant à "relocaliser les biens et les personnes" suite aux conclusions du rapport du député Cousin, remis au gouvernement fin 2011 ou de la nouvelle stratégie nationale de gestion intégrée du trait de côte.

Les pouvoirs publics attendent les conclusions qui seront tirées, en 2014, par le groupement en charge de l'étude (Artelia et partenaires associés). Mais déjà, le fait qu'un tel investissement (950000 euros, payé par l'Europe et l'Etat) soit réalisé pour une étude audacieuse est une preuve du tournant pris récemment en matière de gestion du trait de côte face aux risques d'inondations. Avec la prise en compte récente des effets du changement climatique sur les côtes (voir le cinquième rapport du GIEC), la décennie 2010 en France ouvre sans conteste une nouvelle approche des problèmes, intégrant les dynamiques littorales et anticipant l'évolution des changements climatiques, comme le prouvent les suites données au rapport du parlementaire de la Manche précité, consacré à la gestion du trait de côte en France : mise en place d'une nouvelle génération de PAPI littoraux (Plan d'Actions et de Prévention des Risques), sortis conjointement à l'appel à projets dit "Cousin", incitant les collectivités à proposer des stratégies intégrant la possibilité de reculs maitrisés, à l'image de la Région Languedoc-Roussillon (HEURTEFEUX et al., 2012) ; initiatives auxquelles le Syndicat Mixte Baie de Somme Grand Littoral Picard a répondu courant 2012.

De la façon dont seront menés les projets qui en découleront dépendra leur réussite et leur acceptation par la population. L'intégration de celle-ci aux projets, en plus d'un grand travail de pédagogie, permettra une nouvelle gouvernance intégrant l'élément marin plutôt que le combattant, avec les atouts tant économiques, écologiques que sociaux que cela pourra engendrer. Il faudra aussi pour cela que les corps d'ingénieurs intègrent cette nouvelle approche qui consiste à accompagner la dynamique côtière davantage que la contrecarrer. Y sont-ils aidés ?

\section{Références bibliographiques}

ARTELIA (2013). Étude de faisabilité : dépoldérisation partielle et éventuelle des BasChamps du Vimeu. La recherche d'un avenir sur un territoire pérenne. Atelier 2, Cayeux-sur-Mer, 14 janvier 2013. http://www.baiedesomme.org/magazine/contenus/2013-0114publication_4882013-01-16.pdf 
BAWEDIN V. (2004). La dépoldérisation, composante d'une gestion intégrée des espaces littoraux ? Prospective sur le littoral picard et analyse à la lumière de quelques expériences: Baie des Veys (Normandie), Aber de Crozon (Bretagne), Tollesbury (Essex) et Freiston Shore (Lincolnshire).Cahiers Nantais, $\mathrm{n}^{\circ}$ 61, pp 11-20.

BAWEDIN V. (2012). Les politiques de retour programmé de la mer : renaturation ou gestion des risques ? Exemples girondins et picards. In BATTIAU-QUENEY Y., LAGEAT Y. et PRAT M-C. L'Homme et la dynamique littorale : maîtrise ou adaptation? Dynamiques environnementales, $n^{\circ}$ 30/2012 - LGPA Editions, Presses Universitaires de Bordeaux, pp 105-115.

BAWEDIN V. (2013). L'acceptation de l'élément marin dans la gestion du trait de côte: une nouvelle gouvernance face au risque de submersion ? Annales de Géographie, n 692, A. Colin, pp 422-444

BAWEDIN V., HOEBLICH J.-M. (2006). Les bas-champs de Cayeux (Somme, France) : vers une gestion intégrée ? Enjeux et perspectives de l'ouverture à la mer d'un espace jusque là protégé. VertigO, Vol. 7, n 3, http://dx.doi.org/10.4000/vertigo.1910

DOLIQUE F., ANTHONY E. (1999). Influence à moyen terme (10-100 ans) d'un estran sableux macrotidal sur la stabilité d'un cordon de galets : la flèche de Cayeux (Picardie, France). Géomorphologie : relief, processus, environnement, $\mathrm{n}^{\circ} 1$, pp 23-38. GOELDNER L. (1999). Réouverture de polders et restauration des marais salés en Angleterre : une technique paradoxale de défense contre la mer. Revue Géographique de Lyon, Vol 74, 1999-1, pp 77-85.

GOELDNER-GIANELLA L. (2008). Dépoldériser en Europe occidentale : les apports d'une géographie sociale de l'environnement à l'étude du milieu littoral. HDR, Université Paris 1, 348 p.

HEURTEFEUX H., SAUBOUA P., LANZELLOTTI P. (2012). Evaluation de la vulnérabilité du littoral de l'Hérault à la submersion marine. XII ${ }^{\text {èmes }}$ Journées Nationales Génie Côtier - Génie Civil, Cherbourg, 12-14 juin 2012, pp 925-932. http://dx.doi.org/10.5150/jngcgc.2012.101-H

PASKOFF R. (1994). Les littoraux. Impact des aménagements sur leur évolution. Masson, Paris, 256 p.

VEYRET Y. (2004). Géographie des risques naturels en France. De l'aléa à la gestion. Hatier, Paris, $251 \mathrm{p}$. 